\title{
EXISTENCE OF NONTRIVIAL SOLUTIONS OF LINEAR FUNCTIONAL EQUATIONS
}

\author{
GERGELY KISS AND ADRIENN VARGA
}

ABSTRACT. In this paper we study functional equation

$$
\sum_{i=1}^{n} a_{i} f\left(b_{i} x+c_{i} h\right)=0 \quad(x, h \in \mathbb{C})
$$

where $a_{i}, b_{i}, c_{i}$ are fixed complex numbers and $f: \mathbb{C} \rightarrow \mathbb{C}$ is the unknown function. We show, that if there is an $i$ such that $b_{i} / c_{i} \neq b_{j} / c_{j}$ holds for any $1 \leq j \leq n, j \neq i$, the functional equation has a noncontant solution if and only if there are field automorphisms $\phi_{1}, \ldots, \phi_{k}$ of $\mathbb{C}$ such that $\phi_{1} \cdot \ldots \cdot \phi_{k}$ is a solution of the equation.

\section{INTRODUCTION}

Studying linear functional equations with constant coefficients is a topic of interest (see, for example [1] - [9] and the references therein). We investigate the functional equation

$$
\sum_{i=1}^{n} a_{i} f\left(b_{i} x+c_{i} h\right)=0 \quad(x, h \in \mathbb{C})
$$

where $a_{i}, b_{i}, c_{i}$ are fixed complex numbers and $f: \mathbb{C} \rightarrow \mathbb{C}$ is the unknown function. In this paper we shall consider those equations of form (1) for which the solutions are generalized polynomials. By a generalized polynomial we mean a function $f: \mathbb{C} \rightarrow \mathbb{C}$ such that, for a suitable $n$, we have

$$
\Delta_{h_{1}} \ldots \Delta_{h_{n+1}} f(x)=0
$$

for every $h_{1}, \ldots, h_{n+1}, x \in \mathbb{C}$. Here $\Delta_{h}$ denotes the difference operator, $\Delta_{h} f(x)=$ $=f(x+h)-f(x)$. The following result is a special case of [9] Theorem 3.9:

Theorem 1.1. Suppose $a_{i}, b_{i}, c_{i}(i=1, \ldots, n)$ are complex numbers, $a_{i} \neq 0$ and $\left|b_{i}\right|+\left|c_{i}\right| \neq$ 0 for every $i=1, \ldots, n$. If

is true for every $x, h \in \mathbb{C}$ and

$$
\sum_{i=1}^{n} a_{i} f\left(b_{i} x+c_{i} h\right)=0
$$

$$
\text { there is an } i \text { such that } b_{i} / c_{i} \neq b_{j} / c_{j} \text { holds for any } 1 \leq j \leq n, j \neq i \text {, }
$$

then $f$ is a generalized polynomial of degree at most $n-2$.

Our aim is to prove that an equation of form (1) satisfying the condition (2) has a noncontant solution if and only if there are field automorphisms $\phi_{1}, \ldots, \phi_{k}$ of $\mathbb{C}$ such that $\phi_{1} \cdot \ldots \cdot \phi_{k}$ is a solution (Theorem 2.3, Theorem 3.5, Corollary 3.6). This result provides a theoretical possibility to decide the existence of non-constant solutions of equation (1) under (2).

Date: October 24, 2014.

1991 Mathematics Subject Classification. Primary 39B22.

Key words and phrases. Functional equations, field isomorphisms, variety.

Kiss was supported by Hungarian Scientific Foundation grant no. 72655. 
The proof uses results of spectral analysis on discrete Abelian groups [5]. Let $(G, *)$ be an Abelian group, and let $\mathbb{C}^{G}$ denote the linear space of all complex valued functions defined on $G$ equipped with the product topology. By a variety on $G$ we mean a translation invariant closed linear subspace of $\mathbb{C}^{G}$. A nonzero function $m \in \mathbb{C}^{G}$ is called an exponential if $m$ is multiplicative; that is, if $m(x * y)=m(x) \cdot m(y)$ for every $x, y \in G$. If a variety contains an exponential function, then we say that spectral analysis holds in this variety. If spectral analysis holds in every variety on $G$, then we say that spectral analysis holds on $G$.

We shall use the result of [5] stating that spectral analysis holds on a discrete Abelian group $G$ if the cardinality of $G$ is less then $2^{\omega}$ (the cardinality of continuum).

Note that the space of the solutions of (1) is a linear space over $\mathbb{C}$, but not necessary translation invariant. There are important special cases such as

$$
\sum_{i=1}^{n} a_{i} f\left(x+b_{i} h\right)=0
$$

and

$$
\sum_{i=1}^{n} a_{i} f\left(b_{i} x+\left(1-b_{i}\right) h\right)=0,
$$

when the space of solutions is translation invariant. (Clearly, if the numbers $b_{1}, \ldots, b_{n}$ are distinct, the equations (3), (4) satisfy condition (2).) In general, if the points $\left(b_{i}, c_{i}\right) \in \mathbb{C}^{2}$ lie on a line not going through the origin $(0,0)$, then the space of solutions is translation invariant. Any two of this type of equations are equivalent in the sense that they can be transformed into each other using a linear substitution. For instance, the substitution of $x^{\prime}=h$ and $h^{\prime}=x-h$ shows the equivalence of the functional equations (3) and (4). The collinearity of $\left(b_{i}, c_{i}\right)$ is not a necessary condition for the subspace of solutions to be translation invariant (see Proposition 3.10).

\section{SOlutions OF ORDER 1}

A function $f_{k}: \mathbb{C} \rightarrow \mathbb{C}$ is a monomial of degree $k$, if there exists a nonzero, symmetric and $k$-additive function $F_{k}: \mathbb{C}^{k} \rightarrow \mathbb{C}$ such that $f_{k}(x)=F_{k}(x, \ldots, x)$ for every $x \in \mathbb{C}$. It is well-known that a function $f$ is a generalized polynomial of degree $m$ if and only if $f=f_{0}+\sum_{k=1}^{m} f_{k}$, where $f_{0}$ is constant and $f_{k}$ is a monomial of degree $k(k=1, \ldots, m)$.

Lemma 2.1. Suppose that $f=f_{0}+\sum_{k=1}^{m} f_{k}$ is a solution of (1), where $f_{0}$ is constant and $f_{k}$ is a monomial of degree $k(k=1, \ldots, m)$. Then each of $f_{0}, \ldots, f_{m}$ is a solution of (1).

Proof. Since any $k$-additive function has the rational homogeneity property in every coordinate, we have

$$
\sum_{i=1}^{n} a_{i} \cdot\left(f_{0}+r \cdot f_{1}\left(b_{i} x+c_{i} h\right)+\ldots+r^{n} \cdot f_{n}\left(b_{i} x+c_{i} h\right)\right)=0
$$

for every $x, h \in \mathbb{C}, r \in \mathbb{Q}$. Let $x, h$ be fixed. Putting $F_{j}=\sum_{i=1}^{n} a_{i} f_{j}\left(b_{i} x+c_{i} h\right)$ we obtain $\sum_{j=0}^{m} F_{j} \cdot r^{j}=0$ for every $r \in \mathbb{Q}$. Thus $F_{j}=0$ for every $j$, proving that $f_{j}$ is a solution of (1). 
It is easy to see that if $\phi$ is an automorphism of $\mathbb{C}$ then $\phi$ is a solution of (1) if and only if

$$
\sum_{i=1}^{n} a_{i} \phi\left(b_{i}\right)=0 \text { and } \sum_{i=1}^{n} a_{i} \phi\left(c_{i}\right)=0 .
$$

Indeed, we have, for every $x, h \in \mathbb{C}$,

$$
\begin{gathered}
\sum_{i=1}^{n} a_{i} \phi\left(b_{i} x+c_{i} h\right)=\sum_{i=1}^{n} a_{i}\left(\phi\left(b_{i} x\right)+\phi\left(c_{i} h\right)\right)= \\
=\left(\sum_{i=1}^{n} a_{i} \phi\left(b_{i}\right)\right) \phi(x)+\left(\sum_{i=1}^{n} a_{i} \phi\left(c_{i}\right)\right) \phi(h) .
\end{gathered}
$$

The following proposition will be used frequently (see [3, Theorem 14.5.1]).

Proposition 2.2. Let $K \subset \mathbb{C}$ be a finitely generated field and $\phi: K \rightarrow \mathbb{C}$ be an injective homomorphism. Then there exists an automorphism $\psi$ of $\mathbb{C}$ such that $\left.\psi\right|_{K}=\phi$.

Theorem 2.3. There is a nonzero additive solution of (1) if and only if there exists a solution of (1) which is an automorphism $\phi: \mathbb{C} \rightarrow \mathbb{C}$ or, equivalently, an automorphism satisfying (5).

Proof. The 'if' statement is obvious.

Let $f_{1}$ be a nonzero additive solution of $(1)$ and let $d \in \mathbb{C}$ be such that $f_{1}(d) \neq 0$. Put $K=\mathbb{Q}\left(b_{1}, \ldots, b_{n}, c_{1}, \ldots, c_{n}, d\right)$, the extension of $\mathbb{Q}$ by the complex numbers $b_{i}, c_{i}, d$ $(i=1, \ldots, n)$.

We put $K^{*}=\{x \in K: x \neq 0\}$; then $K^{*}$ is an Abelian group under multiplication. Let $V$ denote the set of additive functions $f: K \rightarrow \mathbb{C}$ satisfying

$$
\sum_{i=1}^{n} a_{i} f\left(b_{i} x\right)=0 \text { and } \sum_{i=1}^{n} a_{i} f\left(c_{i} x\right)=0
$$

for every $x \in K$. Let

$$
V^{*}=\left\{\left.f\right|_{K^{*}}: f \in V\right\} .
$$

We prove that $V^{*}$ is a variety on the Abelian group $K^{*}$.

It is clear that $V^{*}$ is a linear space, and it is translation invariant with respect to the multiplication. Indeed, if $f$ is additive and satisfies (6), then $x \rightarrow f(a x)$ is also additive, and also satisfies (6) for every $a \in K^{*}$. Let $g: K^{*} \rightarrow \mathbb{C}$ be a function in the closure of $V^{*}$. Extend $g$ by $g(0)=0$. Let $a, b \in K^{*}$ be such that $a+b$ is also in $K^{*}$, i.e. $a+b \neq 0$. Since $g \in \mathrm{cl} V^{*}$, for every $\varepsilon>0$ there exists $f \in V^{*}$ such that

$$
|f(a)-g(a)|<\varepsilon,|f(b)-g(b)|<\varepsilon \text { and }|f(a+b)-g(a+b)|<\varepsilon .
$$

Now, $f(a)+f(b)=f(a+b)$ because $f$ is additive, so $|g(a+b)-g(a)-g(b)|<3 \varepsilon$. This is true for every $\varepsilon$, therefore $g(a+b)=g(a)+g(b)$. This holds for every $a, b \in K$ with $a, b, a+b \neq 0$ and then, by $g(0)=0$, it follows that $g$ is additive on $K$. A similar argument shows that $g$ satisfies (6). This means that $V$ is closed, and thus $V$ is a variety. Note that $V^{*} \neq\{0\},\left.f_{1}\right|_{K^{*}} \in V^{*}$ and $f_{1}$ is nonzero on $K$.

By [5, Theorem 1], if $G$ is a discrete Abelian group of torsion free rank less than continuum, then harmonic analysis holds on $G$. This means that every nonzero variety on $\mathbb{C}^{G}$ contains an exponential. Since $K^{*}$ is countable, we find that $V^{*}$ contains an exponential. 
Let $\phi$ be an exponential element of $V^{*}$. Then $\phi(x y)=\phi(x) \phi(y)$ for every $x, y \in$ $K^{*}$. If we define $\phi(0)=0$ then, by $\phi \in V^{*}, \phi$ becomes additive on $K$, and thus $\phi$ is a homomorphism from the field $K$ to $\mathbb{C}$. Since $\phi \not \equiv 0$, it must be an injective field homomorphism. Since $\phi \in V^{*}$, we have

$$
0=\sum_{i=1}^{n} a_{i} \phi\left(b_{i} x\right)=\sum_{i=1}^{n} a_{i} \phi\left(b_{i}\right) \phi(x) \text { and } 0=\sum_{i=1}^{n} a_{i} \phi\left(c_{i} x\right)=\sum_{i=1}^{n} a_{i} \phi\left(c_{i}\right) \phi(x)
$$

for every $x \in K^{*}$. This implies $0=\sum_{i=1}^{n} a_{i} \phi\left(b_{i}\right)=\sum_{i=1}^{n} a_{i} \phi\left(c_{i}\right)$. Using Proposition 2.2, $\phi$ can be extended to an automorphism of $\mathbb{C}$. This completes the proof.

Theorem 2.4. Suppose that $a_{1}, \ldots, a_{n}$ are nonzero and $b_{1}, \ldots, b_{n}$ are distinct complex numbers. The following are equivalent:

(i) There is a nonconstant solution of (3).

(ii) There is a solution of (3) which is an automorphism of $\mathbb{C}$.

(iii) $\sum_{i=1}^{n} a_{i}=0$, and there exists an automorphism $\phi: \mathbb{C} \rightarrow \mathbb{C}$ such that

$$
\sum_{i=1}^{n} a_{i} \phi\left(b_{i}\right)=0
$$

Proof. (i) $\Longrightarrow$ (ii): The substitution $h=0$ shows that $\sum_{i=1}^{n} a_{i} f(x)=0$, thus either $\sum_{i=1}^{n} a_{i}=0$ or 0 is the only solution. By (i), we have $\sum_{i=1}^{n} a_{i}=0$. This condition implies that the constant functions are solutions.

Let $f(x)$ be a nonconstant solution. By Theorem 1.1, every solution is a generalized polynomial. If the degree of $f$ is $k$, then for suitable $h_{1}, \ldots, h_{k-1}$, the degree of the function $g=\Delta_{h_{1}} \ldots \Delta_{h_{k-1}} f$ is 1 . Then $g(x)=a(x)+c$, where $a(x)$ is a nonzero additive function and $c$ is a constant. Since $g-c$ is a solution, we may assume that $f$ is a not identically zero additive function. Then, by Theorem 2.3 , there is an automorphism of $\mathbb{C}$ which is a solution of (3).

(ii) $\Longrightarrow$ (iii): If $\phi$ is an automorphism which is a solution of $(3)$, then $\sum_{i=1}^{n} a_{i} \phi(1)=0$ and $\sum_{i=1}^{n} a_{i} \phi\left(b_{i}\right)=0$.

(iii) $\Longrightarrow$ (i): It is clear by the previous theorem.

Remark 2.5. Theorem 2.4 does not hold in the general case, because the existence of a nonzero solution of (1) does not imply the existence of a nonzero additive solution. For example, the equation

$$
f(x+y)+f(x-y)-2 f(x)-2 f(y)=0
$$

is satisfied by $x^{2}$. It is easy to check that every additive solution must be zero.

Proposition 2.6. Suppose $\sum_{i=1}^{n} a_{i}=0$ and $\sum_{i=1}^{n} a_{i} b_{i} \neq 0$. If either

(i) $b_{i} \in \mathbb{Q}(i=1, \ldots, n)$, or

(ii) $a_{i} \in \mathbb{Q}(i=1, \ldots, n)$,

then every solution of $\sum_{i=1}^{n} a_{i} f\left(x+b_{i} h\right)=0$ is constant.

Proof. We may assume that $a_{1}, \ldots, a_{n}$ are nonzero and $b_{1}, \ldots, b_{n}$ are distinct. Indeed, by deleting the terms corresponding to $a_{i}=0$ and adding the terms corresponding identical $b_{i}$ 's neither the conditions, nor the conclusion change. Due to Theorem 2.4, if there is a nonconstant solution, then $\sum_{i=1}^{n} a_{i} \phi\left(b_{i}\right)=0$, where $\phi: \mathbb{Q}\left(b_{i}\right) \rightarrow \mathbb{C}$ is an injective homomorphism.

Assuming (i) and taking into consideration that the identity is the only isomorphism over $\mathbb{Q}$, we find $\sum_{i=1}^{n} a_{i} b_{i}=\sum_{i=1}^{n} a_{i} \phi\left(b_{i}\right)=0$, contradicting the assumption. 
Assuming (ii), again we use the fact that $\phi$ fixes the elements of $\mathbb{Q}$. We obtain

$$
\phi\left(\sum_{i=1}^{n} a_{i} b_{i}\right)=\sum_{i=1}^{n} a_{i} \phi\left(b_{i}\right)=0
$$

and $\sum_{i=1}^{n} a_{i} b_{i}=0$, a contradiction.

\section{SOlutions OF Higher ORDER}

First we need some technical lemmas.

Lemma 3.1. Let $f_{k}(x)=F_{k}(\underbrace{x, \ldots, x}_{k})$ be a monoid, where $F_{k}$ is symmetric and $k$-additive.

Then

$$
k ! F_{k}\left(x_{1}, x_{2}, \ldots, x_{k}\right)=\Delta_{x_{1}} \Delta_{x_{2}} \ldots \Delta_{x_{k}} f_{k}(t)
$$

for every $t \in \mathbb{C}$.

Proof. See [3, §9,Lemma 2].

Lemma 3.2. If $\phi_{1}, \ldots, \phi_{k}$ are distinct injective homomorphisms of the field $K$ into $\mathbb{C}$, then there exists an element $x \in K$ such that $\phi_{i}(x) \neq \phi_{j}(x)$ for every $1 \leq i<j \leq k$.

Proof. We use induction on $k$. For $k=1$ and $k=2$ the statement is clear. Suppose $k>2$ and the statement is true for $k-1$. Let $\phi_{1}, \ldots, \phi_{k}$ be distinct injective homomorphisms. By the induction hypothesis, there exists an $x \in K$ such that $\phi_{1}(x), \phi_{2}(x), \ldots, \phi_{k-1}(x)$ are distinct. If they are different from $\phi_{k}(x)$, then we are done. Suppose for example that $\phi_{1}(x)=\phi_{k}(x)$. Since $\phi_{1} \neq \phi_{k}$, there is an $x^{\prime}$ such that $\phi_{1}\left(x^{\prime}\right) \neq \phi_{k}\left(x^{\prime}\right)$. For every $i<k$ the number of integers $m_{i}$ satisfying $\phi_{i}\left(x+m_{i} x^{\prime}\right)=\phi_{1}\left(x+m_{i} x^{\prime}\right)$ is finite. Thus there remains a suitable element of the form $x+m x^{\prime}$.

Lemma 3.3. Let $\phi_{1}, \ldots, \phi_{m}$ be distinct injective homomorphisms of the field $K$ into $\mathbb{C}$, and let $k$ be a positive integer. Then there exists an element $h \in K$ such that

$$
\prod_{j \in J} \phi_{j}(h) \neq \prod_{j^{\prime} \in J^{\prime}} \phi_{j^{\prime}}(h)
$$

whenever $J$ and $J^{\prime}$ are distinct multisets of the elements $1, \ldots, m$ containing each of $1, \ldots, m$ at most $k$ times.

Proof. Let $x$ be as in Lemma 3.2. For every multiset $J$ let

$$
P_{J}(r)=\prod_{j \in J} \phi_{j}(r-x)=\prod_{j \in J}\left(r-\phi_{j}(x)\right)
$$

for every $r \in \mathbb{Q}$. Then $P_{J}$ is a polynomial of the variable $r \in \mathbb{Q}$. If the multisets $J, J^{\prime}$ are distinct, then the polynomials $P_{J}, P_{J^{\prime}}$ are also distinct, because, by the choice of $x$, the numbers $\phi_{j}(x)(j=1, \ldots, m)$ are distinct, and thus the set of roots of $P_{J}$ with multiplicities is different from that of $P_{J^{\prime}}$.

If the multisets $J, J^{\prime}$ are distinct then $P_{J}(r) \neq P_{J^{\prime}}(r)$ for all but a finite number of $r \in \mathbb{Q}$. Therefore, we can choose an $r \in \mathbb{Q}$ such that all the values $P_{J}(r)$ are distinct as $J$ runs through the possible multisets. Then $h=r-x$ satisfies the requirements.

Lemma 3.4. For every field automorphisms $\phi_{1}, \ldots, \phi_{k}$ of $\mathbb{C}$, the product $\phi_{1} \cdot \ldots \cdot \phi_{k}$ is a solution of (1) if and only if

$$
\sum_{i=1}^{n} a_{i} \prod_{j \in J} \phi_{j}\left(b_{i}\right) \prod_{j^{\prime} \notin J} \phi_{j^{\prime}}\left(c_{i}\right)=0
$$


for every $J \subseteq\{1, \ldots, k\}$.

Proof. Let

$$
H_{J}=\sum_{i=1}^{n} a_{i} \prod_{j \in J} \phi_{j}\left(b_{i}\right) \prod_{j^{\prime} \notin J} \phi_{j^{\prime}}\left(c_{i}\right) \quad(J \subset I) .
$$

$(\Longrightarrow)$ We denote $I=\{1, \ldots k\}$. If $\phi_{1} \cdot \ldots \cdot \phi_{k}$ is a solution then, applying (1) with $x=1$ and $h=y \in \mathbb{C}$ we obtain

$$
\begin{aligned}
0 & =\sum_{i=1}^{n} a_{i} \prod_{j=1}^{k}\left(\phi_{j}\left(b_{i}\right)+\phi_{j}\left(c_{i}\right) \phi_{j}(y)\right)= \\
& =\sum_{i=1}^{n} a_{i} \cdot \sum_{J \subset I} \prod_{j \in J} \phi_{j}\left(b_{i}\right) \prod_{j^{\prime} \notin J} \phi_{j^{\prime}}\left(c_{i}\right) \phi_{j^{\prime}}(y)= \\
& =\sum_{J \subset I}\left(\sum_{i=1}^{n} a_{i} \prod_{j \in J} \phi_{j}\left(b_{i}\right) \prod_{j^{\prime} \notin J} \phi_{j^{\prime}}\left(c_{i}\right)\right) \prod_{j^{\prime} \notin J} \phi_{j^{\prime}}(y)= \\
& =\sum_{J \subset I} H_{J} \prod_{j^{\prime} \notin J} \phi_{j^{\prime}}(y) .
\end{aligned}
$$

We may assume that the automorphisms $\phi_{1}, \ldots, \phi_{m}$ are distinct, and each of $\phi_{m+1}, \ldots, \phi_{k}$ equal one of $\phi_{1}, \ldots, \phi_{m}$. If $\bar{J}$ is a multiset containing the element $i q_{i}$ times $(i=1, \ldots, m)$ then we put $H_{\bar{J}}=H_{J}$, where $J \subset I$ is such that, for every $i=1, \ldots, m$, the cardinality of the set $\left\{j: \phi_{j}=\phi_{i}\right\}$ equals $q_{i}$. Clearly, (8) can be written in the form

$$
\sum_{\bar{J}} n_{\bar{J}} H_{\bar{J}} \prod_{j^{\prime} \notin \bar{J}} \phi_{j^{\prime}}(y)=0,
$$

where $\bar{J}$ runs through the distinct multisets of size at most $k$ consisting of elements $1 \ldots m$, and the coefficient $n_{\bar{J}}$ are suitable positive integers. By Lemma 3.3, we can choose $h$ such that the values of the products of these injective homomorphisms at $h$ are distinct. Then, applying (9) with $y=h, h^{2}, \ldots, h^{N}$, we get the following system with the notation $\prod_{\bar{J}} \phi_{j}(h)=M_{J}$ :

$$
\begin{gathered}
\sum_{\bar{J}} n_{\bar{J}} H_{\bar{J}} \cdot M_{\bar{J}}=0 \\
\sum_{\bar{J}} n_{\bar{J}} H_{\bar{J}} \cdot\left(M_{\bar{J}}\right)^{2}=0 \\
\vdots \\
\sum_{\bar{J}} n_{\bar{J}} H_{\bar{J}} \cdot\left(M_{\bar{J}}\right)^{N}=0 .
\end{gathered}
$$

This is a Vandermonde system which has no nontrivial solution if $N$ is at least the number of multisets $\bar{J}$ with the given properties. This completes the proof of (7). 
$(\Longleftarrow)$ Since $\phi_{j}$ is an automorphism, $\phi_{j}\left(b_{i} x+c_{i} h\right)=\phi_{j}\left(b_{i}\right) \phi_{j}(x)+\phi_{j}\left(c_{i}\right) \phi_{j}(h)$. Thus,

$$
\begin{aligned}
& \sum_{i=1}^{n} a_{i} \prod_{j=1}^{k} \phi_{j}\left(b_{i} x+c_{i} h\right)=\sum_{i=1}^{n} a_{i} \prod_{j=1}^{k}\left(\phi_{j}\left(b_{i}\right) \phi_{j}(x)+\phi_{j}\left(c_{i}\right) \phi_{j}(h)\right)= \\
= & \sum_{i=1}^{n} a_{i} \sum_{J \subseteq\{1, \ldots, k\}} \prod_{j \in J}\left(\phi_{j}\left(b_{i}\right) \phi_{j}(x)\right) \prod_{j^{\prime} \notin J}\left(\phi_{j^{\prime}}\left(c_{i}\right) \phi_{j^{\prime}}(h)\right)= \\
= & \sum_{J \subseteq\{1, \ldots, k\}}\left(\sum_{i=1}^{n} a_{i} \cdot \prod_{j \in J} \phi_{j}\left(b_{i}\right) \prod_{j^{\prime} \notin J} \phi_{j^{\prime}}\left(c_{i}\right)\right) \prod_{j \in J} \phi_{j}(x) \prod_{j^{\prime} \notin J} \phi_{j^{\prime}}(h)=0,
\end{aligned}
$$

because every term is 0 . Thus the product of $\phi_{1} \cdot \ldots \cdot \phi_{k}$ is a solution of (1).

Theorem 3.5. For every positive integer $k$ the following are equivalent.

(i) There exists a monomial of degree $k$ which is a solution of (1).

(ii) There exist field automorphisms $\phi_{1}, \ldots, \phi_{k}$ of $\mathbb{C}$ such that $\phi_{1} \cdot \ldots \cdot \phi_{k}$ is a solution of (1).

(iii) There exist field automorphisms $\phi_{1}, \ldots, \phi_{k}$ of $\mathbb{C}$ such that

$$
\sum_{i=1}^{n} a_{i} \prod_{j \in J} \phi_{j}\left(b_{i}\right) \prod_{j^{\prime} \notin J} \phi_{j^{\prime}}\left(c_{i}\right)=0
$$

for every $J \subseteq\{1, \ldots, k\}$.

Proof. (ii) $\Longrightarrow\left(\right.$ i) is trivial with $f_{k}(x):=\operatorname{sym}\left(\phi_{1} \cdot \ldots \cdot \phi_{k}\right)(x, \ldots, x)$.

(i) $\Longrightarrow$ (ii): Suppose that there is a solution of (1) of degree $k$. By Lemma 2.1, there is a solution which is a monomial of degree $k$. Let $f_{k}(x)=F_{k}(x, \ldots, x)$ be a solution, where $F_{k}$ is nonzero, symmetric and $k$-additive. Let $d_{1}, \ldots, d_{k} \in \mathbb{C}$ be such that $F_{k}\left(d_{1}, \ldots, d_{k}\right) \neq 0$. We put

$$
K=\mathbb{Q}\left(b_{1}, \ldots, b_{n}, c_{1}, \ldots, c_{n}, d_{1}, \ldots, d_{k}\right) .
$$

Let $\left(K^{*}\right)^{k}=\left\{\left(x_{1}, \ldots, x_{k}\right): x_{1}, \ldots, x_{k} \in K \backslash\{0\}\right\}$. Then $\left(K^{*}\right)^{k}$ is an Abelian group under multiplication by coordinates. Since $\left(K^{*}\right)^{k}$ is countable, spectral analysis holds on it according to [5]. Let $V$ be the set of all functions $S: K^{k} \rightarrow \mathbb{C}$ such that $S$ is $k$-additive and the function $x \mapsto S\left(s_{1} x, s_{2} x, \ldots, s_{k} x\right)$ satisfies (1) for every $s_{1}, s_{2}, \ldots s_{k} \in K$. We prove that $\left.F_{k}\right|_{K^{k}} \in V$. By Lemma 3.1,

$$
k ! \cdot F_{k}\left(s_{1} x, s_{2} x, \ldots, s_{k} x\right)=\Delta_{s_{1} x} \Delta_{s_{2} x} \ldots \Delta_{s_{k} x} f_{k}(0)=\sum_{j=1}^{N} \pm f_{k}\left(e_{j} x\right)
$$

with suitable $e_{1}, \ldots e_{N} \in \mathbb{C}$. Thus $F_{k}\left(s_{1} x, s_{2} x, \ldots, s_{k} x\right)$ is a linear combination of functions of the form $f_{k}(e x)$, which are also solutions of (1). It can be easily seen by simultaneous substitutions $x \rightarrow e x$ and $h \rightarrow e h$. Therefore, $F_{k}\left(s_{1} x, s_{2} x, \ldots, s_{k} x\right)$ is a solution, and thus $\left.F_{k}\right|_{K^{k}} \in V$.

We denote

$$
V^{*}=\left\{\left.f\right|_{\left(K^{*}\right)^{k}}: f \in V\right\} .
$$

It is easy to check that $V^{*}$ is a closed linear subspace of $\mathbb{C}^{\left(K^{*}\right)^{k}}$, translation invariant with respect to the multiplication; i.e. it is a variety. Also, $V^{*} \neq\{0\}$, because $\left.F_{k}\right|_{\left(K^{*}\right)^{k}} \in V^{*}$.

Since spectral analysis holds on $V^{*}$, there is an element $B\left(x_{1}, x_{2}, \ldots, x_{k}\right) \in V^{*}$ which is multiplicative in each coordinate. That is,

$$
B\left(x_{1} y_{1}, x_{2} y_{2}, \ldots, x_{k} y_{k}\right)=B\left(x_{1}, x_{2}, \ldots, x_{k}\right) \cdot B\left(y_{1}, y_{2}, \ldots, y_{k}\right)
$$


for every $x_{i}, y_{i} \in K^{*}$. Therefore,

$$
\begin{aligned}
B\left(x_{1}, x_{2}, \ldots, x_{k}\right) & =B\left(x_{1}, 1, \ldots, 1\right) \cdot B\left(1, x_{2}, \ldots, 1\right) \cdot \ldots \cdot B\left(1,1, \ldots, x_{k}\right)= \\
& =\phi_{1}\left(x_{1}\right) \cdot \phi_{2}\left(x_{2}\right) \cdot \ldots \cdot \phi_{k}\left(x_{k}\right)
\end{aligned}
$$

for every $x_{1}, \ldots, x_{k}, \in K^{*}$. We extend $\phi_{j}$ to $K$ by $\phi_{j}(0)=0$. Then each $\phi_{j}$ is an injective homomorphism, because it is additive by the $k$-additivity of $B$, and it is multiplicative as well. Therefore, $\phi_{1}(x) \cdot \ldots \cdot \phi_{k}(x)=B(x, \ldots, x)$ is a solution of (1) on $K$. Using Proposition 2.2, we can extend $\phi_{j}$ to $\mathbb{C}$ as an automorphism. (iii) $\Longleftrightarrow($ ii): It is clear by using Lemma 3.4.

Corollary 3.6. Suppose that equation (1) satisfies condition (2). Then (1) has a nonconstant solution if and only if there is a $k \in \mathbb{N}$ and there are automorphisms $\phi_{1}, \ldots, \phi_{k}$ such that $\phi_{1} \cdot \ldots \cdot \phi_{k}$ is a solution.

Proof. If $f$ is a nonconstant solution then, by Theorem 1.1, it must be a generalized polynomial of degree $k>0$. Then we can apply Theorem 3.5.

Remark 3.7. Condition (2) is necessary in Theorem 1.1 and Corollary 3.6. For instance, it is easy to construct a nonzero solution $f$ to the functional equation $f(x)-f(2 x)=0$ although every generalized polynomial solution of this equation is zero due to the rational homogeneity property. Thus, there are no automorphisms $\phi_{1}, \ldots, \phi_{k}$ such that $\phi_{1} \cdot \ldots \cdot \phi_{k}$ is a solution, because, clearly, the product $\phi_{1} \cdot \ldots \cdot \phi_{k}$ is a generalized polynomial. We note that if the pairs of parameters $\left(b_{i}, c_{i}\right)$ lie on a line going through the origin, then the functional equation can be reduced to the form $\sum_{i=1}^{n} a_{i} f\left(d_{i} x\right)=0$ which has only the identically zero generalized polynomial solution in general.

In the case of functional equations of the form (3) or (4) we can say more than in Theorem 3.5, due to the translation invariance of the space of solutions with respect to the additive structure.

Theorem 3.8. If $\phi_{1} \cdot \ldots \cdot \phi_{k}$ is a solution of the functional equation (3), where $\phi_{i}$ is an automorphism for every $i=1, \ldots, k$, then every subproduct of them is also a solution of (3).

Proof. By Lemma 3.4, $\phi_{1} \cdot \ldots \cdot \phi_{k}$ is solution of (3) if and only if

$$
\sum_{i=1}^{n} a_{i} \phi_{j_{1}}\left(b_{i}\right) \ldots \phi_{j_{s}}\left(b_{i}\right)=0
$$

for every choice of the integers $0 \leq j_{1}<j_{2}<\ldots<j_{s} \leq k(0 \leq s \leq k)$. Therefore, the conditions are automatically satisfied for any subproduct, thus the statement is clear.

Theorem 3.5 motivates the question whether or not the existence of a solution of order $k$ implies the existence of solutions of the form $\phi^{k}$, where $\phi$ is an automorphism of $\mathbb{C}$. The next proposition shows that the answer is negative.

Proposition 3.9. There is a linear functional equation which has a solution of degree two, but has no solution of the form $\phi^{2}$ where $\phi$ is an isomorphism.

Proof. Put $K=\mathbb{Q}(i)$. This field has only 2 isomorphism, $\phi_{1}(z)=z$ and $\phi_{2}(z)=\bar{z}$. Using Theorem 3.5. it is enough to guarantee that:

(1) $\sum_{i=1}^{n} a_{i}=0$, otherwise there is no non-trivial solution.

(2) $\sum_{i=1}^{n} a_{i} b_{i}=0$ and $\sum_{i=1}^{n} a_{i} \overline{b_{i}}=0$ which imply that $\phi_{1}$ and $\phi_{2}$ are solutions of the functional equation. 
(3) $\sum_{i=1}^{n} a_{i} b_{i}^{2} \neq 0, \sum_{i=1}^{n} a_{i} \overline{b_{i}^{2}} \neq 0$ which means that neither $\phi_{1}^{2}$ nor $\phi_{2}^{2}$ is a solution.

(4) $\sum_{i=1}^{n} a_{i}\left|b_{i}\right|^{2}=0$ which implies that $\phi_{1} \phi_{2}$ is a solution.

It can be easily shown that

$$
f(z+1)+f(z-1)-f(z+i)-f(z-i)=0
$$

is such an equation.

In the last proposition we will show that the translation invariance of the space of solutions does not imply that the functional equation can be reduced to the form (3).

Proposition 3.10. There is a functional equation of the type (1) which only has constant solutions, and the pairs $\left(b_{i}, c_{i}\right)$ are not collinear.

Proof. It is easy to find integers $a_{i}, b_{i}$ and $c_{i}(i=1, \ldots, n)$ with the following properties: $\sum_{i=1}^{n} a_{i}=0, \sum_{i=1}^{n} a_{i} b_{i}^{r} \neq 0$ for any $r=1,2, \ldots$ and the pairs $\left(b_{i}, c_{i}\right)$ are not collinear. Since the identity is the only injective homomorphism of $\mathbb{Q}$, it follows from Theorem 3.5 that if there is a nonconstant solution, then there is a solution which is the $m^{\text {th }}$ power of the identity on $\mathbb{Q}$ for a suitable $m \in \mathbb{N}$. Substituting $x=1, h=0$ we get $\sum_{i=1}^{n} a_{i} b_{i}^{m}$ which is nonzero. Thus any solution of this equation must be constant.

Acknowledgements: The authors would like to thank Professor Miklós Laczkovich and Professor László Székelyhidi for their valuable help to prepare this paper.

\section{REFERENCES}

[1] J. A. Baker, A general functional equation and its stability, Proc. Am. Math. Soc. 133 (2004), no. 6, 1657-1664 (2005)

[2] K. Baron and W. Jarczyk, Recent results on functional equations in a single variable, perspectives and open problems, Aequationes Math. 61 (2001), 1-48.

[3] M. Kuczma, An introduction to the Theory of Functional Equations and Inequalities, (1985).

[4] M. Laczkovich, Polynomial mappings on Abelian groups, Aequationes Math. 68 (2004), 177-199

[5] M. Laczkovich and G. Székelyhidi, Harmonic analysis on dicrete abelien groups, Proc. Am. Math. Soc. 133 (2004), no. 6, 1581-1586

[6] S. Mazur and W. Orlicz, Grundlegende Eigenschaften der polynomischen Operationen, Fund. Math. (1939), 42-50.

[7] S. Stević, Behaviour of solutions of some linear functional equations at infinity, Appl. Math. Comput. 219 (2013), 6134-6141.

[8] L. Székelyhidi, Note on exponential polynomials, Pacific J. Math. 103 (1982), 583-587.

[9] L. Székelyhidi, On a class of linear functional equations, Publ. Math. (Debrecen) 29 (1982), 19-28.

E-mail address: kisss@cs.elte.hu

Department of Analysis, Eötvös Loránd University, 1117 Budapest, Pázmány Péter SÉTÁNY 1/C, HungARY

E-mail address: vargaa@eng.unideb.hu

Faculty of Engineering, University of Debrecen, H-4010 Debrecen, Pf. 12, Hungary 